\title{
Beiträge zur Kenntnis der Fauna von Süd-Afrika.
}

\author{
Ergebnisse einer Reise von Prof. Max Weber \\ im Jahre 1894.
}

VII. Lacertilia (Eidechsen).

\author{
Von \\ Dr. Jean Roux, \\ Kustos am Naturhistorischen Museum in Basel.
}

Mit Tafel 14-15.

Unter reichhaltigen zoologischen Ausbeuten brachte Herr Prof. Max Weber von seiner Forschungsreise in Süd-Afrika (1894) eine große Eidechsensammlung mit, die er dem Naturhistorischen Museum in Amsterdam schenkte. Er war so gütig, mir die Bearbeitung dieser schönen Sammlung zu überlassen, wofür ich ihm an dieser Stelle meinen herzlichen Dank aussprechen möchte. Ferner erhielt ich gleichzeitig von demselben Museum andre, zum Teil schon bestimmte süd-afrikanische Lacertilier, die Herr BReYen im Jahre 1896 in Transvaal (Rustenburg und Pretoria District) erbeutet hat. ${ }^{1}$ )

Die Eidechsensammlung von M. Weber stammt aus verschiedenen Regionen von Süd-Afrika. Für weitere Auskunft über die genaue

1) Die WEBER'schen Exemplare werde ich in Folgendem mit einem $\mathrm{W}$ und die BREYER'schen mit einem $\mathrm{B}$ bezeichnen. 
Lage der verschiedenen in dieser Arbeit zitierten Fundorte sowie auch über die geophysischen und zoogeographischen Verhältnisse dieser Gegend verweise ich auf die Arbeit von M. Weber. ${ }^{1}$ )

Die hier bearbeitete Sammlung umfaßt im ganzen 65 Species, den verschiedenen Familien der Lacertilia angehörend, mit der einzigen Ausnahme der Amphisbaenidae. Durch eine beträchtliche Anzahl von meist schön konservierten Individuen sind besonders die Geckonidae, Agamidae, Zonuridae und Lacertidae repräsentiert. Unter diesen 65 Arten erweisen sich 6 als neu, deren ausführliche Beschreibung weiter unten $\mathrm{zu}$ finden ist.

Für eine bessere Kenntnis der geographischen Verbreitung der Eidechsen in Süd-Afrika sowie auch der Verwandtschaft und der Variabilität mancher Arten aus verschiedenen Fundorten ist diese herpetologische Sammlung besonders interessant.

$\mathrm{Zu}$ bestem Dank bin ich Herrn Dr. G. A. Boulenger in London verpflichtet, der mir in einigen zweifelhaften Fällen mit seinen in so liberaler Weise gegebenen Ratschlägen zur Seite stand.

Herrn Dr. F. Römer in Frankfurt a. M. bin ich auch für die freundliche Überlassung von Vergleichsmaterial aus dem SEnckenBERG'schen Museum sehr dankbar.

Die Aufzählung der Species geschieht in der Reihenfolge, wie sie in den Katalogen Boulenger's angeführt ist.

Ich verweise für die vollständige Liste der bis im Jahre 1898 in Süd-Afrika nachgewiesenen Reptilien- und Amphibienarten auf die Arbeit von W. L. Sclater (1898). ${ }^{2}$ )

Seit dieser Arbeit sind die 3 folgenden neuen Eidechsenarten beschrieben worden $:^{1}$ )

Agama distanti BLGR., in: Ann. Mag. nat. Hist. (7), Vol. 9, 1902, p. 339. Ichnotropis longipes BLGR., in: Proc. zool. Soc. London, 1902, Vol. 2, p. 17.

Typhlosaurus cregoi BLGr., in: Ann. Mag. nat. Hist. (7), Vol. 12, 1903, p. 434 .

1) Beiträge zur Kenntnis der Fauna von Süd-Afrika, I. Zur Kenntnis der Süsswasser-Fauna von Süd-Afrika, in: Zool. Jahrb., Vol. 10, Syst., 1898 , p. 135.

2) Siehe Literaturverzeichnis am Schluß.

3) Siehe Nachtrag am Schluß. 


\section{Geckonidae.}

\section{Phyllodactylus porphyreus (DAUD.).}

Cat. Liz., Vol. 1, p. 87.

W. Range Cottage, Tafelberg bei Kapstadt. 1894. 4 Expl. (2 우, 1 ठ, 1 juv.).

W. Knysna, Kapkolonie. Okt. 1894. 2 Expl.

Die beiden letzten Individuen heller gefärbt, einige ziemlich deutliche, feine, braune Flecken auf dem Körper zeigend. Kopf etwas dunkler als der Rumpf. Die Labialen braun gefleckt.

\section{Phyllodactylus lineatus Su.}

Cat. Liz., Vol. 1, p. 92.

W. Büffel-Fluß, Laingsburg. Aug. 1894. 1 Expl. juv.

Im Nest von Otomys oder Gerbillus.

3. Oedura nivaria BLGR.

In: Proc. zool. Soc. London, 1894, p. 608, 726.

W. Durban. 1894. 1 ठ․

B. Transvaal. 1896.1 ठ 1 .

Bei diesen Exemplaren, die ohne Zweifel zu dieser Species gehören, berührt das Rostrale mit seinen obern Ecken die Nasalöffnungen. Das aus Durban stammende Individuum besitzt 16 Poren, das andere nur 12. Die mit Poren besetzten Schuppen sind viereckig, höher als breit und in ihrer hintern Hälfte durchbohrt.

4. Hemidactylus mabuia (MoR. DE JoNnÈs).

Cat. Liz., Vol. 1, p. 122.

B. Pretoria Distr. 1896. 1 q.

\section{Lygodactylus capensis (Su.)}

Cat. Liz., Vol. 1, p. 160.

W. Lourenço Marquès. $1 \mathrm{Expl}$.

W. Verulam, Natal. Nov. 1894. 1 Expl.

B. Pretoria Distr. 1896. 16-Ex.

Alle Tiere stimmen mit der Beschreibung Boulenger's gut über- 
ein, zeigen jedoch am Kinn eine Eigentümlichkeit, die ich hier erwähnen möchte. Das Mentale ist auffallend groß und scheint mit den beiden ersten Unterlabialen verschmolzen zu sein. Es erhält dadurch eine charakteristische Form und zeigt 2 laterale Linien, die von hinten schräg nach vorn konvergieren, ohne sich jedoch zu treffen. Dieselben Verhältnisse sind auch Lygodactylus madagascariensis eigen. ${ }^{1}$ )

\section{Lygodactylus ocellatus n. sp.}

(Taf. 14, Fig. 1, 2, 3.)

\section{B. Pretoria Distr. 1896. 6 Expl. (3 ㅇ․ 3 бठ).}

Kopf eiförmig, länger als breit. Schnauze vorn gerundet, länger als die Distanz zwischen Auge und Tympanum, 2 mal so lang wie der Augendurchmesser. Tympanum sehr klein, rundlich und aufrecht oval. Rostrale breit, nicht so hoch wie bei $L$. capensis, $1^{3} / 4$ bis 2mal so breit wie hoch. Nasalöffnung oberhalb und etwas hinter der Naht zwischen dem Rostrale und dem 1. Supralabiale. Sie ist deutlich von dem Rostrale begrenzt und außerdem noch von 2 Schuppen, von denen die obere größer ist als die andere. (In 2 Fällen habe ich, aber nur auf einer Seite, ein winziges Schüppchen bemerkt, das kaum mit der Nasalöffnung in Berührung stand.) Die 2 vergrößerten Schuppen auf der Schnauze durch ein Körnchen voneinander getrennt.

Lorealgegend leicht konkav. 7-8 Supralabiale; 5-6 Infralabiale. Das Mentale ist sehr breit, subtriangular, hinten mit 2 oder 3 größern Schuppen in Berührung. Es trägt keine Spur von den lateralen Linien, die wir bei $L$. capensis beschrieben haben.

Die vordern Kinnschuppen zahlreicher als bei den letztgenannten Species, groß und verbreitert, die andern nach hinten allmählich kleiner werdend. Rückenschuppen klein, körnig; die der Schnauze etwas größer. Bauchfläche mit großen, hexagonalen, leicht übereinandergehenden Schuppen bedeckt.

Finger ungleich; der innere rudimentär, der 4. verhältnismäßig kürzer als bei $L$. capensis. Diese Verkürzung ist besonders am Hinterglied deutlich ausgeprägt. 3 Paare unterdigitaler Lamellen. Die distale, erweiterte Partie der Finger kürzer als bei $L$. capensis.

1) TorNier hat auch bei Lyg. picturatus Ptrs. Formveränderungen des Mentale konstatiert. Siehe Tornier (1897), p. 22. 
Männchen mit 7 Präanalporen in einer gebogenen Serie (siehe Fig. 3) (bei $L$. capensis dagegen $4-6$ Poren in einer gebrochenen Linie).

Schwanz kürzer als der Körper, niedergedrückt; Schuppen rundlich, nicht oder leicht dachziegelförmig. Die untern Schuppen größer. Färbung (in Spiritus): Mehr oder weniger dunkeloliv, mit zahlreichen rundlichen, weißen Flecken, die schwarz berandet und auf dem Rücken in Längsreihen angeordnet sind. Die Flecken der vertebralen Linie oft länglich oval. Gliedmaßen auch mit einigen solchen Flecken versehen.

Kopf oliv mit spärlichen, winzigen, weißen Flecken, die oft zu Tupfen oder zu Linien vereinigt sind (z. B. zu parallel laufenden Linien vom Auge bis an die Schulter). Labiale und Mentale je mit einem vordern braunen Fleck. Unterfläche grünlich-weiß. Unterseite der Glieder und des Schwanzes mehr gelblich. 2 Junge sind uniform oliv gefärbt. Das größte ठ mißt:

$\begin{array}{lc}\text { Totallänge } & 60 \mathrm{~mm} \\ \text { Körperlänge } & 28,5 \\ \text { Schwanz (erneuert) } & 22 \\ \text { Kopflänge } & 9,5 \\ \text { Kopfbreite } & 6,5 \\ \text { Vorderglied } & 11 \\ \text { Hinterglied } & 15\end{array}$

Lygodactylus ocellatus ist mit Lyg. capensis nahe verwandt, jedoch durch die Zahl und Anordnung der Nasalschuppen, der subdigitalen Lamellen und der Poren sowie auch durch die charakteristische Körperfärbung leicht zu unterscheiden.

\section{Homopholis wahlbergi (Sм.).}

Cat. Liz., Vol. 1, p. 191.

B. Transvaal. 1896. 1 Expl.

Die Dimensionen dieses Exemplars übertreffen die von BouLENGER angegebenen Maße.

Totallänge $190 \mathrm{~mm}$.

\section{Pachydactylus bibroni (Sir.).}

Cat. Liz., Vol. 1, p. 201.

W. Jakhalswater, Kl.-Namaqualand. Sept. 1894. 6 Expl. (3 ad., 3 juv.). 
W. Zwischen Oranje-Fluß und Jakhalswater, Kl-Namaqualand. 15 Expl.

W. Klipfontein, Kl.-Namaqualand. 4 Expl. (1 ad., 3 juv.).

W. Steinkopf, Kl.-Namaqualand. 3 Expl. (1 ad., 2 juv.).

W. Prince Albert Road, Kapkolonie. 2 Expl.

B. Pretoria Distr., Transvaal. 9 Expl. (5 ad., 4 juv.).

Bei den letzten 9 Individuen sind die auf dem Rücken zerstreuten weißen Punkte sehr deutlich, was bei den andern nicht der Fall ist. Die Platten der Kopfoberseite sind in ihrer Größe variabel. Bei den Exemplaren von Pretoria zählt man 6-7 Interorbitalplatten, bei denjenigen von Kl.-Namaqualand nur 4. Die Konkavität dieser Kopfgegend ist nur bei den letztgenannten Tieren gut zu sehen. Die Zahl der Unterdigitallamellen schwankt zwischen 9 und 12.

Die größten Männchen von Kl.-Namaqualand haben eine Totallänge von $175-180 \mathrm{~mm}$.

\section{Pachydactylus capensis (Svr.).}

Cat. Liz., Vol. 1, p. 202.

W. Steinkopf, Kl.-Namaqualand. 1 Expl.

B. Pretoria Distr. 2 Expl.

B. Transvaal. 2 Expl.

Das Individuum von Steinkopf zeigt auf dem Rücken größere, aber undeutliche Flecken. Der braune Streif jederseits des Hinterkopfs ist dagegen gut ausgeprägt.

\section{Pachydactylus weberi n. sp.}

(Taf. 14, Fig. 4 u. 5.)

W. Klipfontein, Kl.-Namaqualand. 4 Expl.

Kopf eiförmig, länger als bei $P$. capensis. Schnauze $1{ }^{1} / 2$ mal so lang wie der Augendurchmesser. Tympanum oval, schief gelegen. Kopf und Körper sehr niedergedrückt. Gliedmaßen mäßig lang. Finger ziemlich schlank, mit einer distalen Erweiterung, deren Unterseite 5-6 Lamellen trägt.

Schwanz niedergedrückt, deutliche Ringe zeigend.

Schnauze zwischen dem Auge und der Nasalöffnung jederseits etwas gewölbt, mit konvexen Körnchen bedeckt, die größer sind als die des Rückens. Oberfläche des Kopfs und dessen Seiten mit ganz 
feinen Körnchen bedeckt, die mit rundlichen Tuberkeln gemischt sind. Diese Kopftuberkeln nie größer als die des Rückens.

Nasorostrale Schilder voneinander getrennt oder vorn nur eine kleine Strecke miteinander in Berührung. Zwischen beiden ein Körnchen. Rostrale $1{ }^{1} /{ }_{2}$ mal so breit wie hoch. Der zwischen dem Nasorostralen befindliche Winkel stumpfer als bei $P$. capensis.

Oberlabiale 9-10 an der Zahl. Das erstere deutlich pentagonal, ebenso hoch oder manchmal höher als breit, stets mit der Nasalöffnung in Berührung, was bei $P$. capensis nicht vorkommt (siehe Fig. 5).

8-9 Unterlabiale. Die 2 vordern Paare breiter als das Mentale. Letzteres mindestens 2 mal so hoch wie breit, nach hinten an Breite etwas abnehmend. Die hintere Seite dieser 5 Schuppen eine fast gerade Linie bildend.

Rücken mit kleinen, unregelmäßigen, sehr schwach konvexen Schüppchen oder Körnchen bedeckt, die pflasterförmig angeordnet sind. Zwischen diesen Schüppchen sehr große, leicht gekielte, ovale Tuberkel in mehr oder weniger regelmäßigen longitudinalen Serien angeordnet.

Sie sind etwas flacher als bei $P$. capensis. Die mediodorsalen Reihen weniger konvex und schwächer gekielt als die lateralen. Auf den Körperseiten sind die Tuberkel rundlicher und zahlreicher; sie verleihen dieser Gegend ein grobgranulöses Aussehen.

Bauchschuppen übereinanderstehend, von vorn nach hinten an Größe zunehmend.

Gliedmaßen mit derselben Beschuppung wie der Rücken, aber mehr Tuberkel zeigend. Schwanz ziemlich lang mit deutlichen Ringen. Schüppchen glatt, klein und in unregelmäßigen Ringen angeordnet. Außerdem größere Tuberkel, auch in ringförmigen Reihen. Diese Tuberkel länglicher und stärker gekielt als die des Rückens. Die auf der obern und den seitlichen Flächen des Schwanzes sichtbaren Ringe sind durch 2-3 Schüppchenreihen voneinander getrennt. Unterseite des Schwanzes mit großen, glatten, hexagonalen, übereinanderstehenden Schuppen versehen; die der Medianreihe breiter als die andern.

Hauptfärbung hellbraun mit dunklern Zeichnungen auf dem Rücken und auf dem Schwanz. 5-6 dorsale, nicht immer regelmäßige Querstreifen; außerdem kleine, zerstreute Flecke. Gliedmaßen und Kopf auch mit kleinen braunen Tupfen.

Kopf heller als der Körper, gelblich gefärbt. Ein dunkler 
Streif von der Nasalöffnung durch das Auge bis oberhalb des Tympanums reichend und dann mit dem gegenüberstehenden Streif auf dem Hinterkopf zusammentreffend und hier eine Wförmige Figur bildend.

Unterseite des Leibs weiß, fleckenlos.

M a Be:

$\begin{array}{lr}\text { Gesamtlänge } & 86 \mathrm{~mm} \\ \text { Kopflänge } & 14 \\ \text { Kopfbreite } & 8 \\ \text { Körperlänge } & 27 \\ \text { Schwanzlänge } & 45\end{array}$

Diese Species ist zwischen $P$. capensis und $P$. formosus zu stellen. Von diesen Arten ist sie durch die Beschuppung des Körpers, die Zahl und Form der Labialen und des Mentale und die Körperzeichnung leicht zu unterscheiden.

\section{Pachydactylus formosus Sur.}

Cat. Liz., Vol. 1, p. 203.

B. Rustenburg, Transvaal. 1 Expl.

B. Transvaal. 10 Expl. (ad. u. juv.).

Durch folgende Charaktere unterscheidet sich nach Boulenger diese Species leicht von $P$. capensis: "the large tubercles strongly keeled and the small scales between them more irregular and the ventrals scales smaller" (1. c., p. 203). Bei den untersuchten Exemplaren konnte ich konstatieren, daß die relativen Proportionen zwischen den Tuberkeln und den Zwischenkörnchen sehr veränderlich sind. Der Unterschied ist größer bei den erwachsenen als bei den jungen Individuen. Diese Variabilität der Tuberkelgröße erstreckt sich auch auf den Kopf. Außerdem sind noch die Variationen in der Zeichnung der verschiedenen Exemplare beträchtliche. Die 4 braunen Querstreifen des Rückens, welche gewöhnlich breiter sind als die Zwischenräume, können durch zahlreiche Streifen ersetzt sein. Die Breite der Zwischenräume übertrifft in diesem Fall diejenige der Querstreifen. Diese sind braun gefärbt und weisen in ihrer Mitte eine weiße Schuppenreihe auf. Letztere meistens nur bei den Jungen deutlich zu sehen. Es bleiben später allein die braunen Querstreifen oder nur isolierte Flecken übrig.

In einer brieflichen Mitteilung erklärte sich Boulenger geneigt, 
seinen $P$. affinis (Boulrnger, 1896) mit $P$. formosus zu vereinigen. Die bei letztgenannter Art konstatierten Variationen scheinen in der Tat die wenigen Unterschiede zwischen den beiden Species noch zu verringern und ihre Vereinigung zu begründen.

\section{Pachydactylus ocellatus (Cuv.).}

Cat. Liz., Vol. 1, p. 205.

W. Umgebung von Kapstadt. Aug. 1894. 1 Expl.

Die Nasorostrale sind miteinander in Berührung.

W. Kapstadt. Juli 1894. 1 juv.

W. Kapstadt, unter Steinen auf dem Löwenkopf. Juli 1894. 2 Expl.

Nasorostrale getrennt. Eine weiße, undeutliche Linie von der Nasalöffnung zum Auge.

W. Matjesfontein, Kapkolonie. 1894. 1 Expl.

(Im Nest von Otomys oder Gerbillus.)

Bei diesem letzten Exemplar ist die Bauchfläche dunkler und braun gefleckt. Die Kehlflecken sind größer und deutlicher. Die Labialen weisen auch dunkle Flecken auf. Die weiße seitliche Schnauzenlinie sehr deutlich. Schnauze ein wenig länger als gewöhnlich.

Bei der typischen Form bilden das Mentale und die 2 vordern Paare der Unterlabialen eine Gruppe von 5 großen Platten. Hier sind nur 3 große Platten vorhanden, weil das 2. Unterlabialenpaar viel kleiner als das 1 . ist.

Die ventralen sind nicht überall gleich, sondern nehmen deutlich von vorn nach hinten an Größe zu.

Da ich nur 1 solches Exemplar zur Untersuchung habe, kann ich nicht feststellen, ob die eben betrachteten, von der gewöhnlichen Form abweichenden Punkte konstant sind.

Ma Be:

$\begin{array}{ll}\text { Kopflänge } & 9,5 \mathrm{~mm} \\ \text { Kopfbreite } & 6 \\ \text { Körperlänge } & 23 \\ \text { Vorderglied } & 10,5 \\ \text { Hinterglied } & 13 \\ \text { Schwanz } & 23\end{array}$




\section{Pachydactylus mariquensis SM.}

Cat. Liz., Vol. 1, p. 207.

W. Ookiep, Kl.-Namaqualand. 1894. 1 Expl.

\section{Rhoptropus ocellatus BLGR.}

Cat. Liz., Vol. 3, p. 490.

W. Klipfontein, Kl.-Namaqualand. Septbr. 1894. 4 Expl. (3 우, 1 ठ ).

Diese Exemplare stimmen mit der Beschreibung von Boulenger vollkommen überein.

\section{Rhoptropus Synonym von Phelsuma?}

Die von Peters zur Unterscheidung dieser beiden Gattungen gegebenen Merkmale sind von verschiedenen Seiten so entkräftet worden, daß die Aufrechterhaltung von Rhoptropus als eigne Gattung in Frage gestellt werden muß. Der Mangel an Material (ich konnte kein Exemplar von Rh. afer besichtigen) machte es mir unmöglich, eine vollständige Lösung zu geben. Ich will mich hier mit der Anführung einiger Bemerkungen begnügen, welche, meiner Ansicht nach, für die Vereinigung beider Gattungen sprechen.

Wie bei Phelsuma sind die Finger von Rhoptropus in ihrer Länge sehr verschieden. Sie zeigen in den Breiteverhältnissen der basalen und distalen Partien keine größern Unterschiede als gewisse Phelsuma-Arten (z. B. Ph. lineatum). Der 4. Finger ist bei Rh. ocellatus wenigstens an der Basis deutlich dünner. Peters wollte noch in der Anwesenheit einer Kralle am Ende der Zehen ein für Rhoptropus charakteristisches Merkmal sehen. BoEtTGer (1894) aber sagt von Rh. afer (p. 88): „Trotz aufmerksamer Betrachtung bin ich nicht imstande, die von Peters erwähnten Krallen zu sehen; ich vermute daher, daß dieselben ganz fehlen." Ich selber bin bei meinen Studien über Rh. ocellatus nicht glücklicher gewesen.

Was die Form der Pupille anbetrifft, so soll diese bei Rhoptropus vertikal sein; bei unsern Individuen ist sie fast oder ganz kreisrund. Solche Unterschiede sind auch in der Gattung Phelsuma nachgewiesen worden. F. MüLler (1887) sagt nämlich von Ph. güntheri (p. 289 u. 290): „Boulenger giebt (char. generis) circuläre Pupille an. Bei unserm Stück ist dieselbe unverkennbar vertical-oval." 
Peters sah ferner bei Rhoptropus keine Femoralporen, weil er wahrscheinlich nur $q \circ$ vor sich hatte. Boulenger dagegen zählte bei 1 of von Rh. ocellatus 31 Poren in derselben Anordnung wie bei Phelsuma.

Thre Anwesenheit konnte ich auch bei dem $\delta$ von Klipfontein konstatieren (30 an der Zahl). Endlich bemerkte ich bei meinen Exemplaren die bei Phelsuma vorkommenden postanalen Spalten.

Die 2 "Gattungen" zeigen dazu noch eine so große Ähnlichkeit in dem Aussehen und der Beschuppung, daß die noch bestehenden Abweichungen nach meiner Ansicht nicht Gattungsunterschiede genannt werden können.

Ich möchte noch hinzufügen, daß bis heute die Rhoptropus-Arten nur im Westen von Süd-Afrika (Damaraland, Mossamedes, Kapstadt, Hereroland, Kl.-Namaqualand) aufgefunden wurden. Ihre Anwesenheit im Osten (Transvaal, Oranje etc.), dessen herpetologische Verhältnisse noch nicht ganz aufgeklärt sind, ist, so viel ich weiß, noch nicht nachgewiesen worden. Sollte aber wirklich eine Lokalisation der Rhoptropus-Arten im Südwesten Afrikas gegenüber einer solchen der Phelsuma-Arten auf der östlichen afrikanischen Küste und benachbarten Inseln (Madagaskar usw.) bestehen, so hätten wir noch keinen genügenden Grund, um zwei so nahestehenden Formen in zwei verschiedenen Gattungen zu erhalten.

Wie oben gesagt, lasse ich jedoch die Frage noch offen. Eine vollständige und genaue vergleichende Studie dieser beiden „Gattungen“ wäre sehr wünschenswert.

\section{Agamidae.}

\section{Agama distanti BLgR.}

In: Ann. Mag. nat. Hist. (7), Vol. 9, 1902, p. 339.

B. Rustenburg, Transvaal. 5 ㅇ grav.

B. Pretoria Distr. 22 Expl. (5 ठठठ, 4 фᄋ, 13 juv.).

B. id. 10 Expl. (1 o, 9 juv.).

Diese mit $A$. hispida (L.) sehr nahe verwandte Art unterscheidet sich nach Boulenger (1905) hauptsächlich durch ihre auffallend große Ohröffnung und den Mangel oder die schwache Entwicklung der Kiele an den Bauchschuppen. Bei einigen Individuen, Jungen und andern, sind jedoch diese Kiele manchmal noch sehr deutlich zu sehen. Bei 
A. hispida sind alle Schuppen der Unterseite sehr stachlig, was bei A. distanti nicht der Fall ist.

Das occipitale Schild ist bei den Jungen verhältnismäßig groß.

Die verbreiterten, gekielten Dorsalschuppen scheinen bei dieser Species zahlreicher und unregelmäßiger in ihrer Anordnung als bei A. hispida zu sein; die andern sind verhältnismäßig sehr klein.

Die Grundfarbe des Rückens und der Bauchseite ist mehr oder weniger dunkelgelb. Kehle mit feinen schwarzen, meist longitudinalen Linien versehen. Der charakteristische, dorsale, gelbe Mittelstreif sehr deutlich; bei einigen Individuen aus voneinander getrennten, länglichen, gelben Flecken bestehend. 10-12 Analporen.

Die Individuen von Rustenburg sind von den andern etwas verschieden. Die Form des Kopfs ist mehr oval, nach hinten ganz wenig verbreitert. Nach der Größe ihres Tympanums, der Anwesenheit der gelben, dorsalen Mittelstreifen gehören sie zu dieser Species. Die vergrößerten, dorsalen, gekielten und stachligen Schuppen sind aber nicht so zahlreich, wie dies bei $A$. distanti gewöhnlich der Fall ist. Sie weisen eine Anordnung in Längsreihen und auffallende Größe auf. Diese Exemplare könnten fast ebensogut als A. hispida mit gelbem Längsstreif gelten. Sie zeigen jedenfalls die zwischen den beiden Arten existierende innige Verwandtschaft.

Die Weber'sche Sammlung enthält keine eigentliche $A$. hispida. Diese Art mit längstem 3. Finger, stark gekielten und stachligen Kinn- und Bauchschuppen scheint nach den meisten von den Autoren angegebenen Fundorten hauptsächlich in der südlichsten Partie der Kapkolonie zu Hause zu sein. Gegen Norden ist sie durch 2 mit ihr sehr verwandte Formen mehr oder weniger ersetzt; diese sind: in Transvaal $A$. distanti und im Norden der Kapkolonie und im Namaqualand die jetzt in Frage kommende Species A. brachyura BLGR.

\section{Agama brachyura BlgR.}

Cat. Liz., Vol. 1, p. 350.

W. Matjesfontein, Kapkolonie. 7 Expl. (3 ðす, 4 우).

W. Steinkopf, Kl.-Namaqualand. 3 Expl. (2 ठठ, 1 juv.).

W. Zwischen Oranje-Fluß und Jakhalswater. 6 Expl. $(2$ ठठ 1 \&, 3 juv.).

Die Individuen von Matjesfontein sind dunkler gefärbt als die von Jakhalswater. Die länglichen weißen Flecken der Dorsalmittellinie sind sehr deutlich und dunkelbraun umrandet. 
Die Exemplare von Kl.-Namaqualand haben auf dem Rücken eine gelbe bis ziegelrote Grundfarbe. Die paarigen schwarzen Flecken sind sehr deutlich zu sehen. Die Bekielung der Bauchund der Kinnschuppen ist bei den meisten Exemplaren noch angedeutet, bei einigen sogar ziemlich stark. Die für $A$. hispida charakteristischen Schuppenstacheln sind hier nur bei 2-3 Expl. auf den Seiten der Kehle und der Brust schwach entwickelt.

Bei allen Individuen ist die 4. Zehe etwas länger als die 3.

Die dorsalen, vergrößerten, stark gekielten Schuppen sind mehr oder weniger in Längsreihen oder ganz unregelmäßig angeordnet.

Präanalporen 11 an der Zahl. Der Körper ist namentlich bei den 우 sehr niedergedrückt.

Schwanz ist seiner Länge variabel. Maß des längsten ठ $223 \mathrm{~mm}$.

Diese Form wurde schon in Damaraland als $A$. hispida nachgewiesen [Boettger (1887), p. 142].

\section{Agama aculeata MerR.}

Cat. Liz., Vol. 1, p. 351.

W. Oranje-Fluß, Kl.-Namaqualand. $18 \mathrm{Expl}$ (ad. 4 ठठठ์, 4 qक; juv. 7 ठठठ, 3 우).

W. Jakhalswater, Kl.-Namaqualand. 1894. 1 đ juv.

Das Occipitale ist bei den jüngern Individuen verhältnismäßig größer als bei den erwachsenen Exemplaren. Die Schilder der Kopfoberseite sind auffallend groß und subäqual, oft leicht gekielt.

12-14 Analporen in einer nach hinten gebrochenen Linie.

\section{Agama armata Ptrs.}

Cat. Liz., Vol. 1, p. 352.

W. Ladysmith. 1894. 1 q grav.

Die Totallänge dieses Individuums beträgt $140 \mathrm{~mm}$.

\section{Agama atra DAUD.}

Cat. Liz., Vol. 1, p. 352.

W. Knysna, Kapkolonie. Okt. 1894. 1 q.

Fast uniform grau gefärbt. Schwanzflecke sehr deutlich.

W. Zwischen Oranje-Fluß und Jakhalswater, Kl.-Namaqualand. 4 ठठ. 
Totallänge des größern đ̊ $267 \mathrm{~mm}$.

W. Steinkopf, Kl.-Namaqualand. 4 ठठす, 1 q.

Das größte ơ mißt $285 \mathrm{~mm}$.

Diese Maße übertreffen die Angaben im Katalog Boulenger's um ziemlich viel.

W. Klipfontein, Kl.-Namaqualand. 1894. 1 juv.

W. Steinkopf, Kl.-Namaqualand. 1894. 4 Expl. (3 ad., 1 juv.).

W. Jakhalswater, Kl.-Namaqualand. 1894. 1 q juv.

W. Matjesfontein, Kapkolonie. Aug. 1894. 9 Expl. (2 우, 3 むð, 2 juv., 2 pull.).

W. Mossel-Bai, Kapkolonie. 21. Okt. 1894. 1 o juv.

W. Kapstadt, Kapkolonie. Juli 1894. 1 ठ juv.

W. Franschhoek, Kapkolonie. Aug. 1894. 1 q juv.

Die Reihe der Präanalporen zählt von 12-16 Poren, die in einer gekrümmten Linie angeordnet sind.

Die meisten Exemplare von Kl.-Namaqualand haben eine sehr deutliche, mehr oder weniger breite, gelbe Mittellinie auf dem Rücken, die mit vergrößerten glatten Schuppen bedeckt ist. Die Bauchfläche und die Kehle sind schwarz.

Einige Individuen von Steinkopf sind mehr gelblich und zeigen deutliche, paarig angeordnete, schwarze Flecken auf dem Rücken und dem Schwanz, etwa wie bei gewissen $A$. aculeata. Sie weisen im allgemeinen kleinere und zahlreichere Körperschuppen auf als die schwarz gefärbte Form, und die vergrößerten, zerstreuten Schuppen sind auf den ersten Blick fast nicht zu sehen.

Vielleicht sind diese Exemplare als eine besondere Varietät zu betrachten, die man als micropholis bezeichnen könnte und zu welcher die früher beschriebenen $A$. micropholis Мтсніе. und $A$. microterolepis BLGR. zu rechnen wären.

\section{Agama atricollis SM,}

Cat. Liz., Vol. 1, p. 358.

W. Lower Illovo, Natal. 2 qя.

B. Distr. Waterberg, Transvaal. 1896. 1 ठ

Länge $300 \mathrm{~mm}$.

B. Pretoria Distr. 9 Expl. (3 ㅇ, 3 ㅇ juv., 3 ठठ juv.).

B. Rustenburg, Transvaal. 1896. 1 ð.

Außerdem brachte Prof. M. Weber von Mossambique 1 Exemplar von 
20bis. Agama mossambica Pтrs.

1 ช.

\section{Zonuridae.}

21. Zonurus cataphractus (BOIE).

Cat. Liz., Vol. 2, p. 255. 1 juv.

W. Zwischen Orange-Fluß und Jakhalswater, Kl.-Namaqualand.

\section{Zonurus cordylus (L.).}

Cat. Liz., Vol. 2, p. 256.

W. Kapstadt. Juli 1894. 2 Ex. (1 ad., 1 juv.).

W. Range Cottage, Tafelberg b. Kapstadt. 4. Aug. 1894. 26 Expl. (13 ad., 13 juv.).

W. Franschhoek, Kapkolonie. Aug. 1894. 1 ad.

W. Knysna, Kapkolonie. Okt. 1894. 36 Ex. (14 ad., 22 juv.).

Die Exemplare von Knysna und von Franschhoek sind braun bis braunrot gefärbt, mit zerstreuten hellern Flecken. Bei diesen typischen Individuen weist das Frontonasale immer eine sechsseitige Form auf. Fast überall zeigt das Frontale eine vordere mediane Furche, die bis zur Mitte oder manchmal auch weiter nach hinten reicht. Die aus Range Cottage und Kapstadt stammenden Exemplare sind uniform schwarz gefärbt; ihr Frontale zeigt keine mittlere Furche.

In der Form der Frontonasale wurden mehrere Variationen beobachtet, die die innige Verwandtschaft dieser Species namentlich mit $Z$. vittifer aufs klarste beweisen. Diese Variationen wurden schon von BARBoza du Bocage (1895) und Boettger (1899) konstatiert.

Die zwei seitlichen Suturen der hexagonalen Frontonasale sind manchmal sehr kurz. Bei 2 Individuen fehlen sie sogar ganz. In diesem Fall kommt daher das Nasale jederseits mit dem Präfrontale in Berührung, das viereckig gewordene Frontonasale vollkommen einschließend. Bei 1 Exemplar ist dieses Verhältnis nur auf einer Seite zu sehen.

Bei 2 jungen Individuen bildet das hexagonale Nasofrontale mit dem Frontale eine Naht, welche die zwei Präfrontale voneinander trennt. 3 Stück von Knysna (1 ad., 2 juv.) besitzen ein sechsseitiges

Zool. Jahrb. XXV. Abt. f. Syst. 
Frontonasale, welches nach hinten mit einem kleinern, hexagonalen unpaaren präfrontalen Schild in Berührung kommt und die beiden eigentlichen Präfrontale vollständig trennt.

Nach Matschie (1891) kann eine solche supplementäre Platte auch bei $Z$. vittifer vorkommen.

\section{Zonurus cordylus var. vittifer (REICHN.) Roux emend.}

Syn.: Zonurus vittifer Reichnow, in: Zool. Anz., 1887, p. 372.

W. Jasper don. Süd-Afrika. 1 ad.

W. Ladysmith, Natal. 4 Expl. (3 ad., 1 juv.).

B. Pretoria Distr. 12 Expl. (7 ad., 5 juv.).

Nur 1 Exemplar lag Reichnow für seine Beschreibung von $Z$. vittifer zugrunde. TonNien (1897) gab später eine neue vollständigere Diagnose dieser Art nach demselben Individuum, zeigt aber zugleich für andere mit Recht als $Z$. vittifer bestimmte Exemplare des Berliner Museums die große Variabilität von gewissen Charakteren, die Reichnow als typisch für seine Art gehalten hatte. Der Berliner Herpetologe hebt die innige Verwandtschaft dieser Species hervor. Nur kleine Unterschiede bestehen noch zwischen den beiden Arten. Wie er auch gezeigt hat, existieren sogar Zwischenformen, die für das Aufgeben von $Z$. vittifer als eigentliche Art und für seine Vereinigung mit $Z$. cordylus sprechen.

$\mathrm{Zu}$ ganz ähnlichen Resultaten bin ich, wie man weiter unten sehen wird, auch gekommen nach Prüfung des Materials, das mir zur Verfügung stand. Deshalb stelle ich jetzt den frühern Z.vittifer von Reichnow als Varietät zu Z. cordylus.

Bei den untersuchten Exemplaren scheint in der Anordnung der großen Kopfplatten ein Punkt konstant zu sein; es ist die Trennung des Rostrale von dem Frontonasale durch die Nasalia. Das aus dem typisch hexagonalen viereckig gewordene Frontonasale variiert noch in seiner Form und in seinen Dimensionen, was die Distanz zwischen diesem Schild und dem Frontale beeinflußt.

Was das Loreale anbetrifft, konnte ich feststellen, daß die von Reichenow als typisch für seine Art angegebene Abwesenheit nur zufällig ist. Das Loreale existiert bei manchen Individuen und zeigt auch große Variationen in seinen Dimensionen. Dieses Schild kann groß, hoch, trapezoid sein und so das Nasale von dem Präoculare trennen; manchmal aber ist es kleiner, dreieckig. In diesem Fall bilden oben die genannten Schilder eine kürzere oder längere Sutur 
je nach der Dimension des Zügelschildes. Tonnier (l. c.) führt für die Lorealgegend von $Z$. tropidostemum auch manche Variationen an. Das Frontale variiert ebenso in seiner Form je nach seiner Lage zu den benachbarten Platten. Es kann sechs- oder siebenseitig sein.

Das Interparietale ist manchmal fünfseitig, manchmal viereckig und auch in seiner Form sehr veränderlich. Alle diese konstatierten Variationen beweisen, daß man die Kopfbeschuppung nicht als Artcharakter brauchen kann und daß ihr bisher zu großes Vertrauen geschenkt worden ist.

Bei den untersuchten Exemplaren von $Z$. cordylus var. vittifer sind die Nasalia ganz hinten durchbohrt.

Die Nackenschuppen, die gewöhnlich fast gleichgroß sind, zeigen sehr oft bei den Individuen dieser Varietät eine Eigentümlichkeit. Die Schuppen der zweiten, hinter den Parietalen gelegenen Reihe sind auffallend größer, länger als die übrigen. Auf meinen Wunsch untersuchte Prof. Tornier das Typusexemplar von $Z$. vittifer in dieser Hinsicht und konnte dieses Verhältnis hier auch konstatieren. Bei andern Exemplaren wurde aber nur ein kleiner Unterschied nachgewiesen. Dieses Merkmal habe ich bei allen von mir untersuchten Individuen sehr deutlich beobachtet. Es scheint ziemlich konstant zu sein.

Wie bei $Z$. cordylus typicus sind die lateralen Schuppen ebenso groß wie die dorsalen. Manchmal zeigen die lateralen Bauchschuppen einen deutlichen Kiel.

Die Zahl der transversalen Reihen der Rückenschuppen schwankt zwischen 24-26 (von den Parietalen bis zur Schwanzwurzel). Diejenige der longitudinalen beträgt manchmal 16, manchmal auch 18. Die ventralen Längsreihen weisen an Zahl 14-16 auf. Die Tiere besitzen 7-8 Femoralporen jederseits. Die Schwanzdornen sind lang und schmal, mehr nach hinten gerichtet und fast gar nicht gekrümmt.

Die Färbung unserer Individuen ist folgende: orangegelb oder braun, mit hellern und dunklern, unregelmäßig zerstreuten Flecken. Oft auf der medianen Dorsallinie ein gelber, hinten mehr oder weniger unterbrochner Streif.

Die Färbung der jungen Exemplare ist immer intensiver rotbraun als die der erwachsenen.

Das größte Individuum von Ladysmith hat eine Totallänge von $160 \mathrm{~mm}$. 
Der frühere $Z$. vittifer zeigt also eine innige Verwandtschaft mit $Z$. cordylus. Mit diesem letzten hat es so viele gemeinsame Charaktere, daß nach meiner Ansicht nur Varietätsunterschiede bestehen (Frontonasal viereckig, Schuppen der 2. Nackenreihe gewöhnlich größer als die andern; dorsale Schuppenreihen etwas zahlreicher).

\section{Zonurus jonesi BLGR.}

In: Ann. Mag. nat. Hist. (6), Vol. 7, p. 417.

W. Walree don. Transvaal, Limpopo. 1892. 1 ad.

B. Pretoria Distr. 11 Expl. (9 ad., 2 juv.)

Diese von Boulenger auch nur nach einem Exemplar aufgestellte Art ist mit $Z$. cordylus sehr nahe verwandt. Hier kann man ebenfalls große Variationen in der Kopfbeschuppung konstatieren. In seiner Beschreibung spricht Boulenger (1891) von einem pentagonalen Nasofrontale, das mit dem Frontale in Berührung steht. Dieses Merkmal ist aber nicht konstant. Unter 12 untersuchten Individuen, die ohne Zweifel zu dieser Species gehören, zeigen nur 3 ein pentagonales Nasofrontale; bei allen andern ist dieses Schild viereckig, also wie bei $Z$. cordylus var. vittifer. Das 5-oder nur 4 seitige Interparietale ist auch in seiner Form sehr veränderlich. Das Schild kann sich nach vorn verlängern und verjüngen; es berührt in diesem Falle das Frontoparietale, bald nur in einem Punkt, bald auf eine kleine Strecke. Es kann aber auch mehr quadratisch und gänzlich von den benachbarten Schildern eingeschlossen sein.

Wie oben gezeigt wurde, kann eine Arttrennung nach der Kopfbeschuppung nicht mehr bestehen. Es bleiben nur noch einige Unterschiede zwischen den beiden Zonurus-Arten (cordylus var. und jonesi) übrig, namentlich in der Beschuppung und in der Färbung des Körpers. Aber auch da wird vielleicht das spätere Auffinden von Zwischengliedern die Verwandtschaft dieser Formen noch deutlicher beweisen.

Ich füge hier einige Merkmale hinzu, die ich bei den untersuchten Individuen feststellen konnte und die in mancher Hinsicht mit der Beschreibung von Boulenger übereinstimmen. - Der Kopf ist bei $Z$. jonesi verhältnismäßig länger und schmäler als bei den verwandten Formen. Die Öffnung in den etwas gewölbten Nasalia liegt ein wenig hinter der Mitte des Schildes. Oft ist eine kleine Naht 
zwischen der Öffnung und der hintern Seite der Nasalplatte deutlich zu sehen.

Die Nackenschuppen sind gleich groß. Die stark gekielten dorsalen Schuppen sind in 10-12 Längsreihen angeordnet. Die Zahl der transversalen Reihen (von den Parietalen bis zur Schwanzwurzel) beträgt 21-23. Die lateralen Schuppen sind kleiner als die dorsalen, was bei den andern verwandten Arten bis jetzt nicht konstatiert wurde. Die Längsreihen der ventralen Schuppen sind 12 an der Zahl, und die 2 mittlern Reihen sind breiter als die andern.

$Z$. jonesi besitzt stachlige, ziemlich stark nach außen gekrümmte Schwanzdornen (etwa wie bei $Z$. giganteus).

Die Zahl der Femoralporen beträgt 5-6 jederseits.

Was die Färbung anbetrifft, ist diese Art dunkelbraun mit schwarzen Flecken, welche auf der Dorsalseite mehr oder weniger in Längsreihen angeordnet sind. Jederseits läuft ein auffallender, für die Species sehr typischer, schwarzer Streif. Am Ohr beginnend, nimmt er nach hinten an Breite zu, besonders oberhalb des Vorderbeins und geht, sich dann verjüngend, bis zum Hinterglied.

\section{Zonurus polyzonus (Sv.).}

Cat. Liz., Vol. 2, p. 257.

W. Matjesfontein, Kapkolonie. Aug. 1894. 2 Expl. (1 ad., 1 juv.).

W. Steinkopf, Kl.-Namaqualand. 3 Expl. (1 ad., 2 juv.). 6 juv.).

W. Klipfontein, Kl.-Namaqualand. Sept. 94. 10 Expl. (4 ad.,

W. Jakhalswater, Kl.-Namaqualand. Sept. 94. 1 Expl.

W. Zwischen Oranje Riv. und Jakhalswater. 5 Expl.

Bei den jungen Exemplaren ist die Schnauze verhältnismäßig kürzer als bei den erwachsenen Individuen. Die Naht zwischen den Präfrontalen ist auch bei ihnen sehr kurz. Die dorsalen, schwarzen Flecken, von denen die jederseits der Medianlinie gelegenen größer sind als die andern, sind oft in 4 Längsspitzen angeordnet. Die Zwischenräume sind manchmal gelb gefärbt. 
Bestimmungstabelle der Zonurus-Arten (mit Zugrundelegung von Boulenger's Tabelle.)

I. Frontonasale mit dem Rostrale in Berührung. Hinterkopf mit großen Dornen Hinterkopf ohne Dornen

II. Frontonasale von dem Rostrale getrennt

A. Körperseiten mit ähnlichen Schuppen wie der Rücken bedeckt

a) Kein Supranasale. Unteraugenlid undurchsichtig

1. Nasale deutlich gewölbt, halbkreisförmig, im Zentrum durchbohrt. Temporaldornen vorhanden Z. cataphractus

2. Nasale nicht oder sehr undeutlich gewölbt, hinter der Mitte oder ganz hinten durchbohrt. Keine Temporaldornen

* Dorsale und laterale Schuppen gleich groß

† Schuppen der 2. Nackenserie nicht größer als die andern: Frontonasale meist sechsseitig

Z. cordylus typicus

†† Schuppen der 2. Nackenserie etwas größer als die andern; Frontonasale meist vierseitig

Z. cordylus vittifer

** Laterale Schuppen kleiner als die dorsalen $Z$. jonesi

b) Ein Supranasal vorhanden. Nasalia sehr klein. Unteraugenlid mit einer durchsichtigen Scheibe

1. Vordere Kinnschuppen glatt, mäßig groß Z. polyzonus

2. Vordere Kinnschuppen beinahe körnig $\quad Z$. pustulatus

B. Seiten mit körnigen Schuppen bedeckt. $\quad Z$. capensis

26. Pseudocordylus microlepidotus (Cuv.).

Cat. Liz., Vol. 2, p. 259.

B. Pretoria Distr. 1896. 3 Expl.

27. Chamaesaura aenea (WIEGM.).

Cat. Liz., Vol. 2, p. 263.

W. Transvaal. 3 Expl.

B. Transvaal. 1 Expl. 


\section{Chamaesaura anguina (L.).}

Cat. Liz., Vol. 2, p. 264.

W. Knysna, Kapkolonie. 1 Expl.

W. Range Cottage, Tafelberg (unter Steinen). 4. Aug. 1894. 2 Expl. (1 ad., 1 juv.).

Das Exemplar von Knysna besitzt monodactyle Gliedmaßen, von denen nur die vordern mit einer Klaue versehen sind. Das Tier ist hellbraun, mit spärlichen, kleinen, schwarzen Flecken. Es zeigt 26 Schuppen rings um den Körper.

Die Individuen von Range Cottage sind dunkler, mit 2 longitudinalen braunen Dorsalstreifen. Bei dem erwachsenen Exemplar sind alle Glieder mit Klauen versehen. Die monodactylen Hinterfüße besitzen je 1 Klaue. Das linke Vorderglied ist ebenfalls monodactyl, besitzt nur 1 Klaue, während das rechte Vorderglied 2fingerig ist und mit 2 deutlichen Klauen versehen ist.

Dieses Exemplar hat 2 Femoralporen und 26 Schuppen rings um den Körper. Sie ist vielleicht als eine zu Ch. didactyla BLGR. (1890, p. 82) führende Form zu betrachten.

Das Junge, von derselben Färbung, zeigt monodactyle mit je einer Klaue versehene Gliedmaßen. Die Zahl der Schuppen um den Körper herum beträgt 24 .

Diese Individuen zeigen in der Form einiger Kopfplatten Variationen, sodaß diesem Merkmal nicht zu viel systematischer Wert beizulegen ist.

Länge der Individuen von Range Cottage: ad. $375 \mathrm{~mm}$, juv. $295 \mathrm{~mm}$.

\section{Varanidae.}

\section{Varanus albigularis (DAUD.).}

Cat. Liz., Vol. 2, p. 307.

W. Lower Illovo, Natal. $3 \mathrm{Expl}$ ( 2 ठఫす, 1 juv.).

B. Transvaal. 1 juv.

Nur die Kopfschilder sind glatt; die dorsalen Schuppen wie die caudalen sind in der Mitte mit einer ovalen, stumpfen Erhebung versehen.

Die Exemplare weisen folgende Zeichnung auf: Grundfarbe gelblich-grau. Jederseits des Nackens einen schwarzen Streif, ober- 
halb des Ohrs beginnend, dann an Breite zunehmend und bis an die Schulter horizontal laufend. Da biegt sich der Streif nach hinten und endet vor dem Arm.

Auf dem Rücken 5 (bei dem juv.) oder 6 (bei den adulten) breite, dunkle, quergestellte Streifen, breiter als die hellern Zwischenräume. Diese letzten nur bei dem juv. von Transvaal mit rundlichen, quergestellten, hellen, dunkel berandeten Flecken versehen.

Die dunklen Streifen teilen sich jederseits in 2 divergierende Linien, die bis auf die Bauchfläche reichen. Der letzte Streif liegt auf der Kreuzgegend, und von den divergierenden, seitlichen Linien liegt die eine vor dem Bein, die andere hinter demselben.

Auf dem Schwanz 6-7 schwarze ringförmige Flecken, die auf der obern Seite besonders breit sind. Die 4 vordern teilen sich seitlich in der gleichen Weise wie diejenigen der Körperseiten. Bei den Jungen ist diese Teilung in 2 Ringe vollständig.

Die Gliedmaßen sind graubraun, mit kleinen, zerstreuten, gelben Flecken, welche bei dem juv. zahlreicher und deutlicher sind. Bauchfläche gelblich-grau, mit grobmaschigem, schwarzem Netz. Kehlfläche manchmal mit einem zentralen, grauschwarzen Fleck versehen (albigularis!)

Ma Be.

Exemplare aus Lower Illovo (Natal).

$\begin{array}{lcl}\text { ` } & \text { Totallänge } & 900 \mathrm{~mm} \\ \text { ` } & " & 850 \\ \text { juv. } & " & 26 \tilde{}\end{array}$

30. Varanus niloticus (L.).

Cat. Liz., Vol. 2, p. 317.

W. Lower Illovo, Natal. 5 Expl. (2 ठす, 3 juv.)

W. Verulam, Natal. 1 万.

Das größte ơ Exemplar von Lower Illovo hat eine Totallänge von $130 \mathrm{~cm}$. 


\section{Lacertidae.}

\section{Nucras tessellata (Sir.).}

Cat. Liz., Vol. 3, p. 52.

W. Jakhalswater, Kl.-Namaqualand. Sept. 1894. 2 Expl.

B. Rustenburg, Transvaal. 1896. 1 juv.

B. Pretoria Distr., Transvaal. 7 Expl. (5 juv.).

B. Transvaal. 2 Expl.

Die aus Transvaal stammenden Jungen haben einen schön orangegefärbten Schwanz. 1 Exemplar besitzt auf einer Seite 2 Postnasale, auf der andern aber nur ein einziges.

Die hellen Streifen des Rückens sowie auch die hellen seitlichen Punkte sind sehr deutlich zu sehen.

32. Nucras delalandi (M. EDw.).

Cat. Liz., Vol. 3, p. 53.

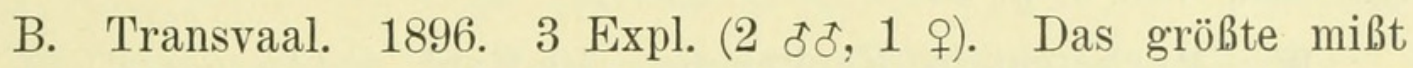
$30 \mathrm{~cm}$.

B. Transvaal. 2 juv.

Die ersten hier zitierten Exemplare weisen nur 1 Postnasale auf. Von den 2 Jungen zeigt das eine 1 Postnasale; das andere besitzt 2 solche auf der einen und nur 1 einziges auf der andern Seite. Eins von diesen beiden juv. ist mit 19 Poren versehen.

33. Ichnotropis capensis ( $\mathrm{Sм}$.$) .$

Cat. Liz., Vol. 3, p. 78.

B. Transvaal. 1896. 2 Expl.

B. Rustenburg, Transvaal. 1896. 3 Expl.

34. Ichnotropis squamulosa PTRS.

Cat. Liz., Vol. 3, p. 79.

B. Rustenburg, Transvaal. 1896. 1 juv.

35. Eremias suborbitalis PтRs.

Cat. Liz., Vol. 3, p. 90.

W. Matjesfontein, Kapkolonie. Aug. 1894. 1 juv.

W. Oranje-Fluß, Kl.-Namaqualand. Sept. 1894. 6 Expl. 
W. Steinkopf, Kl.-Namaqualand. 1894. 16 Expl.

W. Jakhalswater. Sept. 1894. 29 Expl.

Die beiden das 3. Keimplattenpaar bildenden Schilder sind bei manchen Exemplaren durch kleine Schuppen voneinander getrennt. Der vordere Rand der Ohröffnung besitzt vertikal verlängerte Schuppen und 3-4 Lobuli.

\section{Eremias namaquensis D. B.}

Cat. Liz., Vol. 3, p. 91.

W. Jakhalswater, Kl.-Namaqualand. Sept. 1894. 1 Expl.

W. Ookiep, Kl.-Namaqualand. 1 Expl.

W. Steinkopf, Kl.-Namaqualand. 4 Expl.

Die Tibiaschuppen sind manchmal obtus gekielt.

\section{Eremias pulchella GraY.}

Cat. Liz., Vol. 3, p. 93.

W. Matjesfontein, Kapkolonie. 10 Expl.

W. Steinkopf, Kl.-Namaqualand. 1 Expl.

W. Klipfontein, Kl.-Namaqualand. Sept. 1894. 1 Expl.

Bei diesem letzten Exemplar sind die 2 dorsalen schwarzen Streifen sehr breit, sinuös und in den seichten Vertiefungen nach der Außenseite mit einigen weißen Ocellen versehen. Die Körperseiten sind mit großen blauen Ocellen besetzt, die in einem schwarzen Band eingebettet sind. Dieser schwarze Streif verjüngt sich nach vorn und läuft oberhalb des Ohrs bis an den Kopf. Die obern Kopfplatten sind mit schwarzen Flecken versehen.

\section{Eremias lineo-ocellata D. B.}

Cat. Liz., Vol. 3, p. 94.

B. Transvaal. 2 Expl.

Bei dem einen Exemplar sind alle dorsalen Schuppen deutlich gekielt. Das andere besitzt deutlich rugöse Kopfplatten und 3 supplementäre Plättchen, die von dem Frontale, dem Nasofrontale und den Präfrontalen eingeschlossen werden. 


\section{Eremias burchelli D. B.}

Cat. Liz., Vol. 3, p. 95.

W. Matjesfontein, Kapkolonie. 3 Expl. (2 ad., 1 juv.).

Bei einem erwachsenen Exemplar ist der Körnchenkranz um die Suboculare herum vollständig; bei den andern hört er auf der hintern Partie der Innenseite auf, sodaß in dieser Gegend das hintere Suboculare das Frontoparietale berührt.

\section{Eremias capensis (Sмг.).}

Cat. Liz., Vol. 3, p. 96.

W. Steinkopf, Kl.-Namaqualand. 3 Expl.

W. Jakhalswater, Kl.-Namaqualand. Sept. 1894. 1 juv.

\section{Eremias inornata n. sp.}

(Taf. 15, Fig. 1, 2, 3.)

W. Oranje-Fluß, Kl.-Namaqualand. 8 Expl. ( 5 ad.; 3 juv.).

Diese Tiere, die in Amsterdam als $E$. undata aff. bestimmt wurden, zeigen von dieser Species und von der naheverwandten Art $E$. namaquensis bedeutende Abweichungen und stellen sicherlich eine neue Form dar, die zwischen den beiden obengenannten Species einzufügen ist.

Kopfbeschuppung im allgemeinen derjenigen von E. namaquensis ähnlich, doch in einigen Punkten abweichend. Frontonasale mit einer deutlichen, medianen Einsenkung versehen, wie die vordere Partie des Frontale. Distanz zwischen dem Loreale und dem ersten Supraoculare bei allen Individuen genau die Breite des letztgenannten Schildes betragend, 2-3 Schüppchen auf dieser Strecke. Gewöhnlich eine Körnchenserie zwischen dem vordern Supraoculare und den Supraciliaren; 2 solche Reihen zwischen dem hintern Supraoculare und den Supraciliaren. Ohröffnung so hoch wie die Augenöffnung, oben eine längliche, vergrößerte Schuppe aufweisend; 3-4 schwach hervortretende Lobuli.

Unteraugenlid äußerst typisch. Es ist mit einer großen, durchsichtigen Scheibe versehen, die aus $4-5$ ungleichgroßen Platten besteht. Die obern Platten sind größer als die untern. Von diesen letzten kann sich die hintere teilen. Die Grenzlinien zwischen den Platten schwarz gefärbt. 
Das Suboculare ist sehr groß und trifft den Oberlippenrand zwischen der 5 . und 6 . Oberlabialen, welche beide länger sind als die übrigen (ausnahmsweise zwischen der 6. und 7. in einem Fall). Jederseits eine deutliche Falte vom Ohr bis zur Schulter.

Halsband leicht gezähnelt mit 11-13 Platten versehen, deren mittlere oft vergrößert sind: Dorsalschuppen wie bei $E$.namaquensis.

72-75 Schuppen um den Körper herum. Die Schilder der 3 vordern Kinnplattenpaare miteinander in Kontakt (ausnahmsweise nur vorn zusammenstoßend oder - bei 1 juv. - gänzlich durch Schüppchen voneinander getrennt). Ventralplatten in 10 longitudinalen Reihen; die medianen und die äußern sind länger als breit, die andern so breit wie lang oder etwas breiter als lang. 29-31 transversale Reihen. Präanalplatten unregelmäßig gelegen, die 5, 6 oder 7 centralen deutlich vergrößert.

Die Hintergliedmaße, nach vorn gelegt, reicht mit seiner Spitze bis an das Ohr oder zwischen Ohr und Auge. Gliedmaßenbeschuppung wie bei $E$. undata, nur sind die obern Schenkelschuppen deutlich aber stumpf gekielt, ebenso auch manchmal die Oberarmschuppen. 12-14 Femoralporen.

Schwanz mehr als 2 mal so lang wie Kopf und Körper zusammen. Beschuppung wie bei E. namaquensis.

Färbung bei allen Individuen gleich, uniform graubräunlich auf dem Rücken. Auf den Seiten nur einige weißliche Ocellen in einer Linie zwischen den vordern und den Hintergliedmaßen. Körperseiten manchmal auch etwas weißgrau marmoriert. Kopf graubraun. Suboculare und benachbarte Oberlabiale mit einem deutlichen, grauschwarzen Fleck. Gliedmaßen wie der Rücken, spärliche Ocellen auf der Hinterpartie der Schenkel. Finger und Zehen heller. Bauchseite weißlich bis hellgrau. Unterseite der Hinterglieder und des Schwanzes mehr gelblich.

Maße :

Totallänge

Kopflänge

Kopfbreite

Distanz Schnauze-After

Vorderglied

Hinterglied

Schwanz
8

175

14

7

53

21,5

38

122 q grav.

168

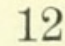

7

49

19,5

36

119 
Diese neue Art unterscheidet sich E. namaquensis hauptsächlich durch ihre 10 Reihen von Ventralplatten und von E. undata u. a. durch die typische Gestalt der Augenscheibe und die Färbung des Körpers.

\section{Scapteira knoxi (M. EDw.).}

Cat. Liz., Vol. 3, p. 109.

W. Matjesfontein, Kapkolonie. 8 Expl.

W. Ebene bei Dieprivier. Juli 1894. 1 Expl.

W. Steinkopf, Kl.-Namaqual. 7 Expl.

W. Ookiep, Kl.-Namaqual. 2 Expl. (1 ad., 1 juv.).

2 Individuen von Matjesfontein haben ein longitudinal geteiltes Nasofrontale.

\section{Gerrhosauridae.}

43. Gerrhosaurus flavigularis WIEGM.

Cat. Liz., Vol. 3, p. 122.

W. Lower Illovo, Natal. 1 Expl.

B. Transvaal. 1 juv.

Das Exemplar von Lower Illovo zeigt ungekielte Seitenschuppen; die Präfrontalia sind miteinander in Kontakt, eine kurze Naht bildend. Bei dem Jungen sind alle Schuppen deutlich gekielt, und das Frontonasale ist mit dem Frontonasale in Berührung. Beide Individuen besitzen 13 Femoralporen.

Wie Tornier [1900, p. 593] glaube ich auch, daß G. nigrolineatus HaLL. nur eine Varietät von G. flavigularis darstellt.

\section{Gerrhosaurus typicus (Sм.).}

Cat. Liz., Vol. 3, p. 123.

W. Klipfontein, Kl.-Namaqualand. Sept. 1894. 2 Expl.

\section{Tetradactylus seps (L.).}

Cat. Liz., Vol. 3, p. 124.

W. Knysna, im Walde; Kapkolonie. 1 juv. 


\section{Tetradactylus breyeri n. sp.}

(Taf. 14, Fig. 6).

B. Transvaal. 1 Expl.

Körper schlangenartig. Gliedmaßen sehr kurz und abgeplattet, die vordern 2 fingrig und mit deutlichen Klauen versehen. Der 2. Finger nur winzig entwickelt. Hintergliedmaßen etwas plumper und ohne Klaue; an ihrer verbreiterten Basis 2 Schenkelporen. Man zählt 10 Schuppen an der Vordergliedmaße und 7 solche an der hintern. Dorsal- und Caudalschuppen wie bei den andern Species gekielt und gerippt. Die dorsalen sind in 12 longitudinalen und in 70 transversalen Reihen angeordnet. Die ventralen bilden 8 longitudinale Serien. Präanal- und Postanalschuppen vergrößert. Schwanz sehr lang, vom Rumpf nicht abgesetzt.

Braun gefärbt, jederseits eine schmale, dunkle Linie, vom Auge bis zur Vordergliedmaße reichend. Ein seitlicher, ebenso schmaler Streif beginnt an der Stelle, wo der erstere aufhört, aber etwas mehr dorsalwärts und läuft über den ganzen Körper und den Schwanz. Die laterale Falte stark ausgebildet; sie trägt vorn, auf der Nackenseite, vertikale, schwarze Streifen. Unterseite des Körpers grau grünlich.

Das Tier habe ich zu dieser Gattung gerechnet trotz einiger Besonderheiten in der Kopfbeschuppung, die ich ausführlich erwähnen will.

In seiner Gattungsdiagnose von Tetradactylus sagt Boulenger (Cat. Liz., Vol. 3, S. 124): „Nostril pierced between the firth labial and two nasals." Bei diesem Exemplar ist die Nasalöffnung vom 1. Labiale weit entfernt und nur von 2 Nasalia umrandet. Von dem Rostrale ist sie ebenfalls deutlich getrennt. Da ich nur dieses Individuum vor Augen gehabt habe, kann ich nicht entscheiden, ob das nur ein isolierter Fall ist, oder ob ich es hier mit einem konstanten Charakter einer speziellen Gattung zu tun habe. Bei dieser Familie kann man, wie auch bei den Zonuridae, in der Kopfbeschuppung manche Variationen konstatieren. ${ }^{1}$ )

Frontonasale mit dem Rostrale nicht in Berührung, mit dem Frontale aber eine breite Naht bildend. Frontale länglich, fünfseitig. Die vordere Seite etwas konvex; hinten zwei kurze Seiten, an die

1) Siehe G. Tornier, Die Crocodile, Schildkröten und Eidechsen in Togo, in: Arch. Naturg., 1901, Vol. 67, 1, p. 76 u. 77. 
zwei kleine Frontoparietale stoßen. Zwischen letztern ein längliches, ovales Interparietale, das nach vorn mit der Hinterspitze des Frontale in Berührung steht. Parietalia sehr groß. 4 Supraoculare, das 2. am breitesten. Die 3 ersten mit dem Frontale in Kontakt. Auf einer Seite 4, auf der andern 5 Supraciliaria, das 2. am längsten. Suboculare sehr lang und schmal. 2 niedere Supralabiale vor diesem Schild, ein höheres hinten. 5 Temporalia $(2+2+1)$, das letzte sehr groß. 3 Unterlabiale, das 2. besonders eng. Hinter dem Mentale die Schilder der 2 andern Kinnschuppenpaare miteinander in Kontakt. Schwanz eine vollständige Drehung um die Längsachse zeigend von rechts nach links (natürliche Erscheinung?).

Ma Be:

$\begin{array}{lc}\text { Totallänge } & 222 \mathrm{~mm} \\ \text { Kopf und Körper } & 56 \\ \text { Schwanz } & 166 \\ \text { Kopflänge } & 8 \\ \text { Kopfbreite } & 4,5 \\ \text { Vorderglied } & 4,5 \\ \text { Hinterglied } & 4\end{array}$

\section{Scincidae.}

47. Mabuia gruetsneri (Ptrs.).

Cat. Liz., Vol. 3, p. 171.

B. Pretoria Distr. 1 Expl.

B. Transvaal. 5 Expl.

Ich rechne zu dieser Species diese 6 aus Transvaal stammenden Exemplare, die jedoch mit der im Boulenger'schen Kataloge gegebenen Beschreibung nicht in allen Punkten übereinstimmen. Diese Individuen gehören zweifellos zu dieser Gruppe und zeigen folgende Hauptmerkmale.

Das 1. Loreale mit dem 1. Labiale nicht im Kontakt (bei einem einzigen Exemplar ausnahmsweise sehr wenig). Bei allen Individuen ist das Rostrale von dem Nasofrontale durch die Subnasalia getrennt. Das Nasofrontale bildet mit dem Frontale eine kurze Naht, aber nur bei 4 von den untersuchten Individuen; die 2 andern besitzen zwischen den eben genannten Schildern zusammenstoßende 
Präfrontalia. Das Frontale ist mit dem 2. und dem 3. Suboculare in Berührung (nur in einem Fall auch mit dem 1.). Das 2. Suboculare ist das größte und berührt fast immer das Präfrontale. Frontal ebensolang wie die Frontoparietalia und das Interparietale zusammen. Die Parietalia vollständig durch das Interparietale getrennt, hinten nie eine Naht bildend. Das Suboculare zwischen dem 4. und 5. Oberlabiale den Lippenrand erreichend, unten nicht verengt.

Ohröffinung schief, mit 2-3 undeutlichen, kleinen, runden Lobuli am Vorderrand. Oft die Ohrspalte sehr eng und die Lobuli unter den vom Vorderrand vorspringenden Schuppen gar nicht zu sehen.

Von den 6 Exemplaren besitzt ein einziges 36 Schuppen um den Körper herum, ein anderes hat 35 Schuppen, die 4 andern aber nur 34.

Nacken und Dorsalschuppen mit 3 Kielen versehen. Die Hintergliedmaße, nach vorn gelegt, erreicht mit ihrem Ende das Handgelenk der Vordergliedmaße. Die unterdigitalen Lamellen mit einem einzigen, stark ausgeprägten Kiel besetzt.

Schwanz $1^{1 / 4}$ mal so lang wie Kopf und Körper zusammen. Färbung sehr konstant bei den 6 Exemplaren, von der von Boulenger angegebenen ziemlich abweichend. Wie bei $M$. trivittata besitzt der Rücken 3 mehr oder weniger ausgeprägte helle Streifen, von denen der medio-dorsale der breiteste ist. Die 2 lateralen beginnen oberhalb des Auges. Zwischen diesen Linien schiefgelegene schmale, schwarze Querstreifchen. Diese letzten immer viel schmäler als bei M. trivittata. Seitlich sind die Schuppen grau oder bräunlich und zeigen manchmal weiße Flecken. Keine Spur von den schwarzen Dorsalstreifen, die Boulenger angibt. Die Oberlabialen olivengrün mit einem zentralen weißen Fleck, der manchmal nur auf dem Suboculare entwickelt ist. In andern Fällen können diese weißen Flecken sich berührend, bis an das $\mathrm{Ohr}$ sich fortsetzen. Unterseite grünlich.

Maße.

$\begin{array}{lr}\text { Totallänge } & 136 \mathrm{~mm} \\ \text { Kopf und Körper } & 55 \\ \text { Schwanz } & 81 \\ \text { Kopflänge } & 12 \\ \text { Vorderglied } & 16 \\ \text { Hinterglied } & 22\end{array}$




\section{Mabuia trivittata (CUv.).}

Cat. Liz., Vol. 3, p. 195.

W. Steinkopf, Kl.-Namaqualand. 1 Expl.

W. Klipfontein, Kl.-Namaqualand. 1 Expl.

Dieses letzte Individuum besitzt nur 28 Schuppen um den Körper herum.

W. Franschhoek, Kapkolonie. Aug. 1894. 1 Expl.

W. Knysna, Kapkolonie. Okt. 1894. 18 Expl.

Bei 6 von diesen letzten Stücken ist das Frontale mit dem Frontonasale in Berührung. Die Glieder der größern Individuen sind verhältnismäßig plumper.

B. Pretoria Distr. 2 Expl.

\section{Mabuia occidentalis (PтRs.).}

Cat. Liz., Vol. 3, p. 196.

W. Matjesfontein, Kapkolonie. Aug. 1894. 2 Expl.

29 Schuppen um den Körper herum.

W. Steinkopf, Kl.-Namaqualand. 2 juv.

W. Ibid. 1 Expl.

31 Schuppen.

W. Zwischen Oranje-Fluß und Jakhalswater, Kl.-Namaqualand. 1 Expl.

Totallänge $200 \mathrm{~mm}$.

B. Transvaal. 3 Expl.

B. Rustenburg, Transvaal. 2 Expl.

Totallänge des größten $245 \mathrm{~mm}$. Beide besitzen 34 Schuppen rings um den Körper.

\section{Mabuia varia (Ptrs.).}

Cat. Liz., Vol. 3, p. 202.

W. B. U. de Waal Don, Kapstadt. 1 Expl.

Oben uniform braun gefärbt, unten weißlich. Die Parietalia bilden keine Naht hinter dem Interparietale.

W. v. Walree don, Limpopo 1892. Transvaal.

35 Schuppen rings um den Körper.

W. Lourenço Marquès. 2 Expl.

Nasofrontale und Frontale in Berührung.

Zool. Jahrb. XXV. Abt. f. Syst. 
W. Lower Illovo, Natal. $3 \mathrm{Expl}$.

W. Klipfontein, Kl.-Namaqualand. Sept. 1894. 1 Expl.

W. Steinkopf. 3 Expl.

B. Transvaal. 2 Expl.

\section{Mabuia striata (Ртвs.).}

Cat. Liz., Vol. 3, p. 204. 1 Expl.

W. Zwischen Oranje-Fluß und Jakhalswater, Kl.-Namaquland.

Dieses Exemplar besitzt 4 schmale, schwarze Dorsalstreifen. Die äußern beginnen auf dem 2. Supraoculare, die 2 andern auf dem Nacken.

W. Walree don, Limpopo, Transvaal. 1 Expl.

39 Schuppen um den Körper herum. Suboculare den Lippenrand nicht berührend. Nasofrontale mit dem Frontale nicht in Kontakt. Parietalia linter dem Interparietale keine Naht bildend.

W. Lourenço Marquès. $1 \mathrm{Expl}$.

W. Ladysmith, Natal. 2 Expl.

B. Pretoria Distr. 2 Expl.

B. Transvaal. 8 Expl.

Nur bei 2 von diesen letzten Individuen berührt das Suboculare den Lippenrand nicht.

\section{Mabuia sulcata (PтRs.).}

Cat. Liz., Vol. 3, p. 206.

W. Prince Albert Road, Kapkolonie. Sept. 1894. 1 Expl.

Schwarz gefärbt; Bauchseite etwas heller als der Rücken; 38 Schuppen um den Körper herum. Hinterseite der Extremitäten und des Schwanzes gelblich. Obere Gliedmaßenschuppen mit 3 stark ausgeprägten Kielen versehen.

53. Mabuia acutilabris (Ptrs.).

Cat. Liz., Vol. 3, p. 208.

W. Oranje-Fluß, Kl.-Namaqualand. Sept. 1894. 2 Expl.

Uniform gefärbt. Kinn mit zerstreuten, braunschwarzen Flecken. 


\section{Ablepharus wahlbergi (Sir.).}

Cat. Liz., Vol. 3, p. 350.

W. Ladysmith, Natal. 1 Expl.

W. Durban, Natal, unter Steinen. 1 Expl.

B. Transvaal. 3 Expl.

1 Exemplar mit schwarzen dorsalen Linien: Unterhalb des gelben laterodorsalen Streifs ein mehr oder weniger breites und dunkles Band, das die ganze Körperseite einnimmt.

\section{Herpetosaura mira n. sp.}

(Taf. 14, Fig. 7-8.)

B. Transvaal. 1896. $11 \mathrm{Expl.}(10$ ad., 1 juv.).

Wie Tornier (1902) es schon hervorgehoben hat, ist die Gattung Herpetoseps Blgr. als Synonym von Herpetosaura PTrs. zu betrachten. Der Berliner Herpetologe erwähnt folgende 2 Arten: Herpetosaura anguina Blgr. und $H$. arenicola Ptrs. Letztere wurde von Boulenger der Gattung Scelotes zugeschrieben, gehört aber zu Herpetosaura. Hier möchte ich eine 3. sehr interessante Art beschreiben, die ich als $H$. mira bezeichnen will. Diese Form stimmt in ihren Hauptmerkmalen mit Herpetosaura gut überein (Palatinknochen, Nasalöffnung). In andern Punkten aber weicht diese neue Art von den andern Formen genug ab, um einige Änderungen in der bisher gegebenen Gattungsdiagnose zu motivieren. In der Tat sind bei dieser Species die Gliedmaßen vorhanden; die Ohröffnung ist deutlich zu sehen, was bei den andern Arten nicht der Fall ist. Wahrscheinlich wird man später für diese Gattung wie für die nächststehenden Scelotes, Sepsina etc. intermediäre Formen beschreiben, zwischen den mit 4 Extremitäten versehenen und den fußlosen Arten

Mit Zugrundelegung von Boulenger's und Tornier's Angaben kann man die Gattung Herpetosaura folgendermaßen charakterisieren:

Palatinknochen ${ }^{1}$ ) in der Mittellinie des Gaumens nicht aneinander stoßend. Zähne konisch. Unteraugenlid mit Schuppen besetzt. Ohröffnung sichtbar oder verborgen. Nasalöffnung von dem Rostrale und dem winzigen, ringförmigen Nasale umrandet. Rostrale, Supranasale und 1. Labiale an das Nasale stoßend. Postnasale, Präfrontale

1) Infolge eines "lapsus calami" schreibt Tornier (l. c., p. 104) Parietalknochen statt Palatinknochen. 
und Frontoparietale fehlend. Körper sehr lang, Gliedmaßen mehr oder weniger entwickelt oder gänzlich fehlend.

\section{A usführliche Beschreibung.}

Schnauze abgerundet, nach vorn nicht vorspringend. Rostrale sehr groß, dessen von oben sichtbarer Teil etwas kürzer als die Länge des Frontonasale. Analöffnung schief, oval, genau über der Naht zwischen dem Rostrale und dem 1. Labiale gelegen. Die Öffnung ist von dem Rostrale und einem winzigen ringförmigen Nasale umrandet. Die Supranasalia stoßen auf der Mittellinie aneinander und bilden mit dem 1. Labiale eine kurze Naht. Das Supranasale und das 1. Labiale umranden das Nasale. Frontonasale ungefähr 2mal so breit wie lang, mit dem Frontale eine breite Sutur bildend. Frontale so lang oder etwas länger als seine Distanz von dem Schnauzenende; hinten $1 \frac{1}{2}$ mal so breit wie vorn. Interparietale das breiteste Kopfschild, 2mal so breit wie lang; seine Vorderseite konkav. 2 bandförmige Parietalia miteinander in Berührung. 4 Supraoculare, das 2. am breitesten. Die 3 vordern mit dem Frontale in Kontakt. 6 Supralabiale, das 1. am höchsten, das 4. am breitesten und unter dem Auge gelegen. 6 Unterlabiale. Mentale hinten bis zur Hälfte des 1. Supralabiale reichend. Die 3-4 ersten Kinnschuppenpaare etwas größer als die übrigen Ventralschuppen. Temporale Schilder groß. Ohröffnung deutlich sichtbar, oval, kleiner als die Pupille.

Körperschuppen glatt, cycloid, übereinander stehend, überall gleich, in 22 Längsreihen angeordnet.

Gliedmaßen kurz, 5fingerig. Die vordern ungefähr halb so lang wie die hintern. Nach vorn gelegt nimmt die vordere Gliedmaße die Hälfte oder ein wenig mehr als die Distanz zwischen Schulter und Ohr ein. Jeder Finger mit einer deutlichen Klaue versehen. Der 3. Finger am längsten, 2. und 4. etwas kürzer, gleich lang; 1. und 5. sehr klein.

Die hintern Gliedmaßen ebenfalls 5fingerig, genau so lang wie die Distanz zwischen Auge und Vordergliedbasis. Die 4. Zehe am längsten, die 3. etwas kürzer; die 2. halb so lang wie die 4.; 5. länger als 1. Die beiden letztern schwach entwickelt. Alle Zehen ebenfalls mit deutlichen Klauen versehen.

Analschuppen nicht vergrößert. Schwanz lang, vom Rumpf nicht deutlich abgesetzt, mit ähnlichen Schuppen bedeckt wie der Körper, sein Hinterende spitzig. 
Grundfarbe bräunlich, auf dem Bauch mehr gelblich. Jede Schuppenreihe mit longitudinal angeordneten schwarzen Fleckchen, die auf den Körperseiten und auf dem Schwanz etwas größer und ausgeprägter sind als auf dem Rücken. Ventralfläche ebenso mit schwarzen Punktserien besetzt; die Punkte spärlicher auf dem Bauch als auf der Unterseite des Schwanzes.

Kopf dunkler als der Körper. Die Zentralpartie der Schilder dunkelgrau oder schwarz; die Suturlinien heller, bräunlich; sehr deutlich.

Oberseite der Gliedmaßen auch mit schwarzen Punkten versehen. Nach unten sind sie etwas kantig; gelblich gefärbt.

Grundfärbung des Schwanzes in seiner hintern Hälfte manchmal grau statt bräunlich, was auch bei dem einzigen jungen Exemplar der Fall ist.

$$
\text { M a Be: }
$$

$\begin{array}{lc}\text { Totallänge } & 157 \mathrm{~mm} \\ \text { Körperlänge } & 63 \\ \text { Schwanzlänge } & 87 \\ \text { Kopflänge } & 7 \\ \text { Kopfbreite } & 5 \\ \text { Vorderglied } & 6 \\ \text { Hinterglied } & 12,5\end{array}$

Bestimmungstabelle der Herpetosaura-Arten.

I. Gliedmaßen vorhanden

H. mira n. sp.

II. Gliedmaßen fehlend

a) 4 Supraocularia, 6 Supraciliaria

H. anguina BLGR.

b) 3 Supraocularia, 5 Supraciliaria

H. arenicola PTRs.

\section{Sepsina weberi n. sp.}

(Taf. 14, Fig. 9.)

W. Steinkopf, Kl.-Namaqualand. 1 Expl.

Schnauze abgerundet, vorn über den Lippenrand etwas vorspringend. Auge mäßig groß; Unteraugenlid mit Schuppen bedeckt. Rostrale groß; die von oben sichtbare Partie fast gleich lang wie die Hälfte der Länge des Frontale. Supranasalia eine breite Sutur hinter dem Rostrale miteinander bildend. Postnasale ziemlich klein, hinten breiter als vorn. Nasofrontale sehr grob, $1^{3}{ }_{4}$ mal so breit wie lang. Frontale etwas länger als seine Distanz vom Schnauzenende; hinten 3mal so breit wie vorn. Dieses Schild ist mit den 4 Supraocularen in Berührung. Interparietal breiter als lang, mit 
dem 4. Supraoculare jederseits eine kleine Strecke in Kontakt. Seine Länge $2 / 3$ der Frontallänge. Parietalia länglich, bandförmig, eine breite Naht hinter dem Interparietale bildend. Temporalia groß. 6 Oberlabialia. Das 1. am höchsten, die Nasalöffnung berührend. Das 4. am breitesten, unter dem Auge gelegen. 6 Unterlabialia. Die erstern Kinnschuppenpaare größer als die übrigen. Ohröffnung sehr klein, wenig deutlich.

Auf dem Nacken die 3 vordern Schilderpaare größer als die andern. 22 Schuppenserien um den Körper herum, dorsale und ventrale gleich groß. Präanalschuppen verbreitert.

Gliedmaßen kurz, zusammengepreßt, mit je 5 Fingern versehen. Das Vorderglied $3 / 4$ mal so lang wie seine Distanz von der Ohröffnung. Jeder Finger mit einer deutlichen Klaue versehen. Der 3. und 4. Finger gleich lang. Der 2. halb so lang wie die vorigen. 1. und 5. sehr kurz, letzterer etwas länger.

Hintergliedmaßen so lang wie die Distanz zwischen Mundsymphyse und Vordergliedmaßenbasis. Die 4. Zehe am längsten, die 3. fast ebensolang. 5. länger als die 1. Klaue sehr deutlich, ziemlich lang.

Schwanz vom Rumpf nicht deutlich abgesetzt, mit ähnlichen Schuppen wie die des Körpers.

Färbung: Hellbraun; schwarze Flecken auf jeder Schuppenserie, die auf dem Schwanze und auf den Körperseiten deutlicher sind als auf der Ober- und Unterfläche des Körpers. Jederseits eine dorso* lateraler, heller Streif, der auf der Schnauze beginnt; oberhalb des Auges zieht er sich nach hinten, wird undeutlicher auf den Seiten des Körpers und wieder sehr ausgeprägt auf den Schwanzseiten.

Kopfseitenschilder mit schwarzen Flecken. Kinnfläche gelblich. Ventralfläche fast grau. Schwanz etwas dunkler als der Körper. Leider kann ich die genaue Länge des Schwanzes nicht angeben, weil er abgebrochen ist. Er scheint fast ebensolang wie Körper und Kopf zusammen zu sein.

M a Be:

$\begin{array}{lc}\text { Kopf und Körper } & 57 \mathrm{~mm} \\ \text { Schwanz (abgebr.) } & 20 \\ \text { Kopflänge } & 6,5 \\ \text { Kopfbreite } & 4,5 \\ \text { Vorderglied } & 7 \\ \text { Hinterglied } & 12\end{array}$


Diese Species unterscheidet sich von den südafrikanischen Sepsina-Arten u. a. durch ihre 5fingrigen Gliedmaßen.

\section{Acontias meleagris (L.).}

Cat. Liz., Vol. 3, p. 427.

W. Oudshoorn, Kapkolonie. 1 Expl.

W. Knysna, Kapkolonie. Okt. 1894. 3 Expl.

W. Range Cottage u. d. Tafelberg. 1 Expl.

W. Kapstadt. 1 juv.

B. Transvaal. 2 Expl.

\section{Acontias lineatus PTRs.}

Cat. Liz., Vol. 3, p. 428.

W. Matjesfontein, Kapkolonie. Aug. 1894. In der Erde. 1 Expl.

Jede Schuppenserie trägt auf dem Rücken und auf den Seiten einen schwarzen Streif. Dieser löst sich auf der Bauchfläche in getrennte Flecken auf. Die dorsalen Streifen sind am breitesten und am meisten entwickelt; die seitlichen etwas heller und schmäler.

\section{Anelytropidae.}

59. Typhlosaumus lineatus BLGR.

Cat. Liz., Vol. 3, p. 432.

W. Steinkopf, Kl.-Namaqualand. 1 Expl.

Außer den 4 braunschwarzen dorsalen Längsstreifen jederseits die Andeutung einer andern kurzen Linie, hinter dem Auge beginnend und aus kleinen, voneinander getrennten braunen Flecken gebildet.

\section{Chamaeleontidae.}

\section{Chamaeleon quilensis BOCAGE. ${ }^{1}$ )}

Ch. parvilobus BLGR., in: Cat. Liz., Vol. 3, p. 449.

W. Verulam Natal. 1 Expl. ठ.

B. Pretoria Distr., Transvaal. 5 Expl. (2 $q 9,1$ s, 2 juv.).

B. Rustenburg, Transvaal. 5 Expl. (2 우, 3 ठठ).

1) In: Journ. Soc. Lisboa, Vol. 1, 1866, p. 59. 
61. Chamaeleon melanocephalus (GR.).

Cat. Liz., Vol. 3, p. 457.

W. Lower Illovo, Natal. 1 Expl.

62. Chamaeleon caffer BtTGR.

In: Ber. Senckenberg. naturf. Ges. Frankfurt, 1888/89, p. 292.

W. Verulam, Natal. 1 Expl. ㅇ.

Hinterteil des Kopfs nicht erhöht. Die Zahl der typischen Tuberkel der Rückencrista beträgt hier 17, wovon die letzten abgeflacht und fast gar nicht von den andern Schuppen des Rückenfirsts zu unterscheiden sind. Totallänge des Exemplars $112 \mathrm{~mm}$.

63. Chamaeleon pumilus DAUD.

Cat. Liz., Vol. 3, p. 458.

W. Ebene bei Dieprivier, Kap. Juli 1894. 2 Expl.

Schwanz länger als Kopf und Körper zusammen.

64. Chamaeleon damaranus BLGR.

Cat. Liz., Vol. 3, p. 460.

B. Pretoria Distr., Transvaal. 1896. 2 Expl.

Bei diesen Exemplaren decken sich die vordern Kinnlappen kaum. Diese Lappen sind hinten mit einfachen, konischen Tuberkeln gemischt, die allmählich an Größe abnehmen.

65. Chamaeleon namaquensis SM.

Cat. Liz., Vol. 3, p. 462.

W. Jakhalswater, Kl.-Namaqualand. 1894. 1 Expl.

Basel, September 1906. 


\section{Literaturverzeichnis.}

Hier gebe ich eine alphabetisch-chronologisch angeordnete Liste der Arbeiten, die mit der Eidechsenfauna von Süd-Afrika in Beziehung stehen und während und nach der Publikation des weltbekannten und hochgeschätzten Werks von Boulenger, Catalog of Lizards in the British Museum, 3 Bde., 1885-1887 - das uns als Grundlage diente - veröffentlicht wurden.

Ausnahmsweise zitiere ich vorher das frühere, wichtige und prachtvoll illustrierte Werk von A. Sмıтн, Illustration of South Africa, Vol. 3, Reptiles, London 1849.

1895. Barboza Du Bocage, Herpetologie d'Angola et du Congo, ouvrage publié sous les auspices du ministère de la marine et des colonies, Lisbonne 1895, $19 \mathrm{pl}$.

1896. - Sur quelques Reptiles et Batraciens africains provenant du voyage de Monsieur le Dr. Emile Holub, in: Jornal Sc. mat. phys. nat. Lisboa (2), Vol. 4, 1896, p. 115-120.

1896a. - Sur deux Agames d'Angola à écaillure hétérogène, ibid. (2), Vol. 4, 1896, p. 127-130.

1886. Boettger, O., Beiträge für Herpetologie und Malakozoologie Südwest-Afrikas, I. Zur Kenntnis der Fauna von Angra Pequena, in : Ber. Senckenberg. naturf. Ges. Frankfurt, 1886, p. 3-15, tab. 1. II. Zur Kenntniss der Fauna der Wüste Kalahari, ibid., p. 20.

1887. - Z Zweiter Beitrag zur Herpetologie Südwest- und Süd-Afrikas, ibid., 1887 , p. $135-173$, tab. 5.

1889. --, Herpetologische Miscellen, V. Transvaal, VI. Pondoland, ibid., 1889 , p. $286-295$.

1893. —, Eine neue Eidechse aus Süd-West-Afrika, in: Abhandl. Ber. zool. anthropol.-ethnogr. Mus. Dresden 1892/93, No. 5.

1894. - (siehe FleCK), Aufzählung der Arten, p. 88. 
1895. Böttger, O., Zwei neue Reptilien vom Sambesi, in: Zool. Anz., Vol. 18, 1895, p. 62-63.

1887. Boulenger, G. A., On a new Gecko of the genus Chondrodactylus from the Kalahari Desert., in: Proc. zool. Soc. London, 1887, p. 339.

1888. —, On new or little know South African Reptiles, in: Ann. Mag. nat. Hist. (6), Vol. 2, 1888, p. 136-141.

1890. - First report on additions to the Lizard collection in the British Museum (Natur. Hist.), in: Proc. zool. Soc. London, 1890, p. 77-86, $4 \mathrm{pl}$.

1891. - Description of a new Lizard of the Genus Zonurus from the Transvaal, in: Ann. Mag. nat. Hist. (6), Vol. 7, 1891, p. 417.

1892. - Reptilia and Batrachia, in: Appendix to Distant's Naturalists in Transvaal, London 1892, p. 174-176, fig.

1894. - , On a Gecko from South Afrika, in: Proc. zool. Soc. London, 1894, p. 608.

1894a. - Second report on additions to the Lizard collection in the Natural History Collection, ibid., 1894, p. 722-736, 3 pl.

1896. - Descriptions of two new Lizards from the Transvaal, in: Ann. Mag. nat. Hist. (6), Vol. 17, 1896, p. 21-22.

1902. - A new name for the common Agama of the Transvaal, ibid. (7), Vol. 9, 1902, p. 339.

1902a. -, A list of the Fishes, Batrachians and Reptiles collected by Mr. Helioth Darling in Mashonaland, with descriptions of new species, in: Proc. zool. Soc. London, 1902, Vol. 2, p. 13-18, 3 pl.

1903. - , On a collection of Batrachians and Reptiles from the interior of Cape Colony, in: Ann. Mag. nat. Hist. (7), Vol. 12, 1903, p. $215-217,2$ pl.

1903a. -, On new Lizards in the Britisch Museum, ibid. (7), Vol. 12, 1903 , p. $429-435$.

1905. - On a collection of Batrachians and Reptiles made in South Africa by Mr. C. H. B. Grant and presented to the British Museum by Mr. C. D. Rudd, in: Proc. zool. Soc. London, 1905 (Vol. 2), p. $248-255$.

1888. Fischer, J. G., Herpetologische Mittheilungen, in: Jahrb. Hamburg. wiss. Anst., Vol. 5, 1888.

1894. Fleck, Ed., Dr. Eduard Fleck's Reiseausbeute aus Südwest-Afrika, in: Ber. Senckenberg. naturf. Ges. Frankfurt 1894, II. Vorkommen und Lebensweise der Reptilien und Batrachier von Dr. Ed. Fleck, p. 83 ; Aufzählung der Arten von Prof. O. Boettger, p. 88.

1892. GÜNтhER, A., Report on a collection of Reptiles and Batrachians transmitted by Mr. H. H. Johnston from Nyassaland, in: Proc. zool. Soc. London, 1892, p. 555-558, 3 pl.

1895. van Lith DE JeUde, Th. W., On a collection of Reptiles from Transvaal, in: Notes Leyden Mus., Vol. 16, 1895, p. 227-230. 
1891. Matschie, P., Über eine kleine Sammlung von Reptilien und Amphibien aus Süd-Afrika, in: Zool. Jahrb., Vol. 5, Syst., 1891, p. $605-611$.

1899. MocQUARD, F., Reptiles rapportés de l'Afrique australe et centrale par Monsieur Ed. FoA, in: Bull. Mus. Hist. nat. Paris, Vol. 5, 1899, p. $218-219$.

1887. MÜLLER, Fritz, Fünfter Nachtrag zum Katalog der herpetologischen Sammlung des Basler Museums, in: Verh. naturf. Ges. Basel 1887, p. 249.

1896. Perracca, M. G., Rettili ed Amfibi raccolti a Kazungula e sulla strada de Kazungula a Buluwayo dal Rev. Luigi JaLta, in: Boll. Mus. Zool. Anat. comp. Torino, Vol. 11, 1896, No. 255.

1882. Peters, W., Naturwissenschaftliche Reise nach Mossambique, Zool. Amph., Berlin 1882.

1887. Reichenow, A., Neue Wirbeltiere des Zoologischen Museums in Berlin, in: Zool. Anz., Vol. 10, p. 372.

1898. Sclater, W. L., List of Reptiles and Batrachians of South Africa, with descriptions of new species, in: Ann. South African Mus., Vol. 1, 1898, p. 95-112, 1 pl.

1887. Тномпnот, Description de trois espèces nouvelles d'Anolis et d'un Amphisbaenien (Amph. capensis), in: Bull. Soc. philom. Paris (7), Vol. 11, 1887, p. $182-190$.

1897. Tonnier, G., Die Kriechtiere Deutsch-Ost-Afrikas. Beiträge zur Systematik und Descendenzlehre. 5 Taf., 11 Textfig., Berlin.

1900. - Neue Liste der Crocodile, Schildkröten und Eidechsen Deutsch Ost-Afrikas, in: Zool. Jahrb., Vol. 13, Syst., 1900, p. 579-618, 8 Abb.

1902. - Herpetologisch Neues aus Ost-Afrika, in: Zool. Anz., Vol. 25, 1902 , p. $700-704$.

1893. Trimen, R., The South African Vertebrate fauna, in: J. Noble's Illustrated Official Handbook of the Cape and South Africa, London 1893, Reptiles and Batrachians, p. 78-88.

1898. Werner, Fr., Über Reptilien aus Syrien und Süd-Afrika, in: Jahresber. nat. Ver. Magdeburg 1896/98, p. 127-148.

\section{Nachtrag.}

Während des Drucks dieser Arbeit sind folgende Beiträge zur Kenntnis der Eidechsenfauna von Süd-Afrika erschienen:

1906. Boulenger, G. A., Description of a new Chameleon of the genus Rhampholeon from Mashonaland, in: Ann. Mag. nat. Hist. (7), Vol. 18, p. $346-347,1$ Fig. (Rh. marshalli n. sp.). 
1907. Boulenger, G. A., Descriptions of three new Lizards and a new Frog, discovered by Dr. W. J. Ansorge in Angola, ibid. (7), Vol. 19, p. $212-214$.

1907. - - On a collection of Fishes, Batrachians and Reptiles, made by Mr. S. A. Neave in Rhodesia, North of the Zambesi, with field notes by the collector, in: Mem. Proc. Manchester liter. phil. Soc., Vol. 51, part. 3, No. 12. (Keine neue Species.)

1907. - Descriptions of a new Toad and a new Amphisbaenian from Mashonaland, in: Ann. Mag. nat. Hist. (7), Vol. 20, p. 47-49. Textfiguren und Taf. III. (Chirindia swynnertoni n.g. n. sp. Amphisbaenidarum).

1907. Roux, JEan, Sur quelques Reptiles sud-africains, in: Rev. Suisse Zool., Vol. 15, fasc. 1, p. 75-86, Fig. (Typhlacontias gracilis n. sp. Scincidarum).

\section{Erklärung der Abbildungen.}

\section{Tafe] 14.}

Fig. 1. Lygodactylus ocellatus $n . s p$. Dorsalansicht. $2: 1$.

Fig. 2. Dsgl. Hinterfuß. $6: 1$.

Fig. 3. Dsgl. Analgegend. $6: 1$.

Fig. 4. Pachydactylus weberi $n$. sp. Dorsalansicht. 2:1.

Fig. 5. Dsgl. Kopf, von der Seite gesehen. $3: 1$.

Fig. 6. Tetradactylus breyeri $n$. sp. Seitenansicht. $3: 1$.

Fig. 7. Herpetosaura mira n. sp. Dorsalansicht. $2: 1$.

Fig. 8. Dsgl. Kopf, von der Seite gesehen. $4: 1$.

Fig. 9. Sepsina weberi $n$. sp. Seitenansicht. $3: 1$.

\section{Tafel 15 .}

Fig. 1. Eremias inornata n. sp. Dorsalansicht. $2: 1$.

Fig. 2. Dsgl. Kopf, von oben gesehen. $3: 1$.

Fig. 3. Dsgl. Kopf, von der Seite gesehen. $3: 1$. 


\section{$2 \mathrm{BHL}$ Biodiversity Heritage Library}

Roux, J. 1907. "Beiträge zur Kenntnis der Fauna von Süd-Afrika. Ergebnisse einer Reise von Prof. Max Weber im Jahre 1894. VII. Lacertilia (Eidechsen)." Zoologische Jahrbücher 25, 403-444. https://doi.org/10.5962/bhl.part.11960.

View This Item Online: https://www.biodiversitylibrary.org/item/38161

DOI: https://doi.org/10.5962/bhl.part.11960

Permalink: https://www.biodiversitylibrary.org/partpdf/11960

\section{Holding Institution}

MBLWHOI Library

\section{Sponsored by}

MBLWHOI Library

\section{Copyright \& Reuse}

Copyright Status: NOT_IN_COPYRIGHT

This document was created from content at the Biodiversity Heritage Library, the world's largest open access digital library for biodiversity literature and archives. Visit BHL at https://www.biodiversitylibrary.org. 